\title{
Two-Dimensional Silica: Crystalline and Vitreous
}

\author{
M. Heyde, S. Shaikhutdinov, H.-J. Freund \\ Fritz-Haber-Institut der Max-Planck-Gesellschaft, Faradayweg 4-6, 14195 Berlin
}

\begin{abstract}
Two dimensional $\mathrm{SiO}_{2}$ films may be grown on metal single crystal surfaces. It is possible to grow crystalline and vitreous (glassy) films and study their structural, vibrational, and electronic properties. In particular, the structures of a crystalline and a vitreous film may be imaged with atomic resolution side by side which opens avenues to study long standing problems of real space imaging of a crystal to glass transition.
\end{abstract}

\section{Introduction}

Silicon dioxide $\left(\mathrm{SiO}_{2}\right)$ is one of the most widespread materials on our planet and the key material in many technological applications like electronics, sensors, catalysis, and not to forget window glass. Obviously, silica may be crystalline or vitreous. Resolving the atomic structure of silica glass has been an open issue since its proposal in $1932 .{ }^{[1]}$ Crystallinity on the other hand is crucial for electronic properties $^{[2]}$ and as such it is very interesting to study the transition between a crystalline and a vitreous state at atomic resolution. ${ }^{[3]}$ We discuss here the atomic structure of thin silica films which have been grown on metal substrates. ${ }^{[4-8]}$ Such systems are well-suited for a profound application of experimental "surface-science" and computational methods to image the atomic structure of crystalline and amorphous solids as well as to determine electronic and chemical properties of well-defined surfaces. Very interesting relations between the properties of windows glass and a two dimensional layer of only $1 \mathrm{~nm}$ thickness are revealed. ${ }^{[9-11]}$

It is now well established that crystalline silica films may be grown on $\mathrm{Mo}(112)^{[4]}$ and $\mathrm{Ru}(0001)^{[5]}$ in the form of single layers of corner sharing $\left[\mathrm{SiO}_{4}\right]$ tetrahedra with a $\mathrm{SiO}_{2.5}$ composition (the so-called "monolayer" film) as schematically shown in Fig. 1a. 


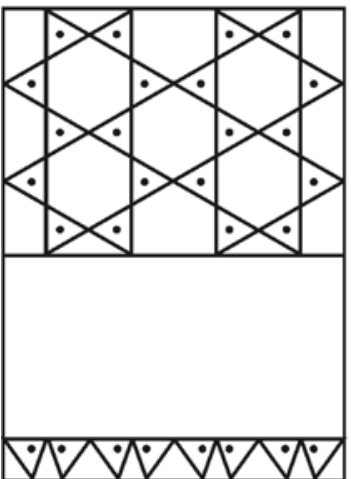

(a)
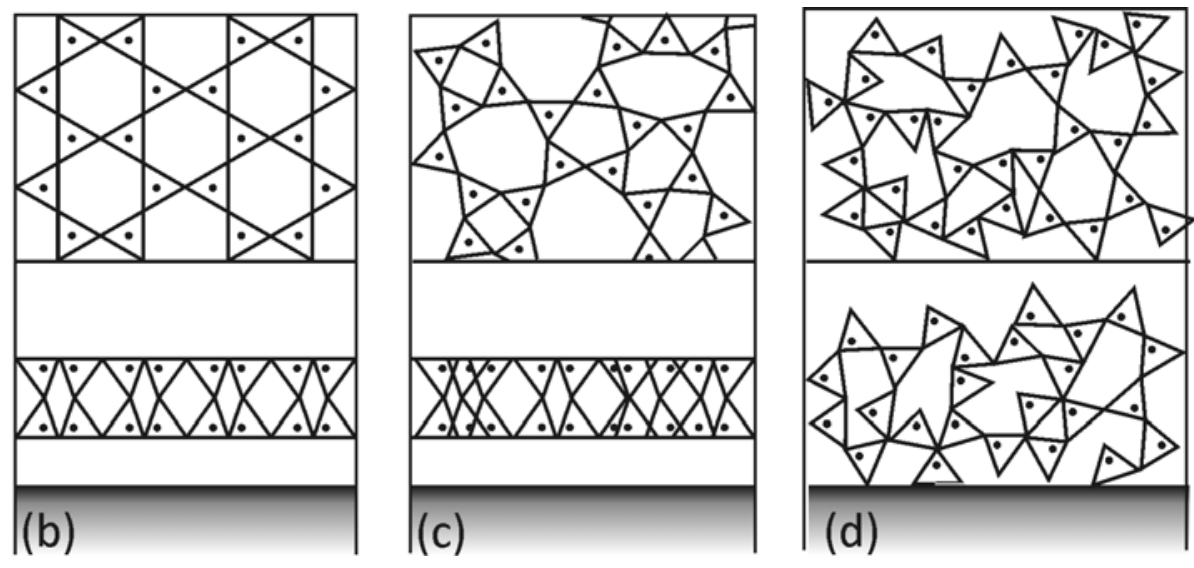

(d)

Figure 1. Schematic representations of silica thin films grown on metal single crystal surfaces. (a-d) Top and cross-sectional views of the monolayer (a) and ordered (b) and disordered (c) bilayer films. (d) Three-dimensional vitreous silica film. All structures are formed by corner-sharing $\left[\mathrm{SiO}_{4}\right]$ tetrahedra. Only the positions of Si are marked by dots, for clarity.

Growth studies on silica films on a Ru(0001) substrate led to the observation of "bilayer" films (see Fig. 1b) in addition to monolayer films. By a combination of infrared reflectionabsorption spectroscopy (IRAS), x-ray photoelectron spectroscopy (XPS), low energy electron diffraction (LEED) and scanning tunnelling microscopy (STM) as well as density functional theory (DFT) calculations, these films were shown to consist of a saturated double layer only bound by dispersion forces to underlying $\mathrm{Ru}(0001)^{[5]}$. In contrast to monolayer films, the bilayer films exist in both, crystalline and vitreous modifications (see Figs. 1b,c). Since the work of Zachariasen in $1932^{[1]}$, who proposed a random network of corner-sharing $\left[\mathrm{SiO}_{4}\right]$ as the atomic arrangement in glass, there was direct convincing experimental verification by imaging missing. ${ }^{[12-15]}$ Now, the study of two-dimensional silica films allows us to directly visualize the atomic structure of the vitreous (or glassy) silica. Our results open a new playground for studying fundamental aspects of glasses such as order-disorder transitions. Attempts to grow multilayer crystalline films on Mo substrates resulted in silica overlayers with smooth, but amorphous surfaces, although the films exhibited phonon spectra very similar to those of the crystalline films obtained on Si single crystal surfaces. Further annealing of the "thick" films at higher temperatures, to increase order, led either to the complete film decomposition or, again, to the monolayer film. ${ }^{[6]}$ 
The results reveal a structural complexity of the silica overlayers on metals, and provide further steps towards our understanding of the atomic structure, structural dynamics and physical and chemical properties of silica.

\section{Silica Films on Ru(0001): Monolayer, Bilayer and Three-Dimensional Structures}

We first address the preparation, growth mode and thermal stability of silica films on $\mathrm{Ru}(0001) .{ }^{[5,16]}$ The preparation includes physical vapor deposition of Si onto the oxygen precovered $\mathrm{Ru}(0001)$ surface in ambient oxygen (typically $10^{-7} \mathrm{mbar}$ ). The presence of chemisorbed oxygen presumably prevents intermixing of $\mathrm{Si}$ and $\mathrm{Ru}$, as judged by XPS, and supplies more reactive, i.e. atomic, oxygen species for oxidation of the Si deposits than molecular oxygen in the gas phase. The full oxidation and film ordering, monitored by LEED, occurs upon high temperature annealing in oxygen (ca. $10^{-6} \mathrm{mbar}$ ) at 1150 - $1200 \mathrm{~K}$ for ca. $5 \mathrm{~min}$. Lowering the substrate temperature during the Si deposition to $100 \mathrm{~K}$ somewhat improves the film quality. Apparently, low temperatures suppress the diffusivity of Si atoms on the surface, thereby favoring the formation of two-dimensional silica sub-oxide structures prior to final oxidation at high temperatures.

At $\mathrm{Si}$ coverage corresponding to a half a monolayer $(\mathrm{ML})$ with respect to $\mathrm{Ru}(0001)-(1 \times 1)$, the surface is almost fully covered by a silica film exposing domains with a honeycomb-like structure with a $\sim 5.5 \AA$ periodicity (see Fig. $2 a$ ), which nicely agrees with the $(2 \times 2)-R u(0001)$ LEED pattern $\left(\mathrm{a}_{\mathrm{Ru}(0001)}=2.71 \AA\right.$ ). The domains are shifted by a half of the lattice with respect to each other, thus producing a network of domain boundaries. The randomly distributed holes are $\sim 1.4 \AA$ in apparent depth. The respective IRA-spectrum shows the principal band at 1134 $\mathrm{cm}^{-1}$ and weaker signals at 1074, 790 and $687 \mathrm{~cm}^{-1}$ (Fig. 2d). This spectrum clearly resembles those observed for $\mathrm{SiO}_{2.5} / \mathrm{Mo}(112)$ films ${ }^{[4,17]}$, were a sharp band at $1060 \mathrm{~cm}^{-1}$, assigned to the stretching vibrations of the Si-O-Mo linkage, dominates the spectra showing also weak signals at 770 and $675 \mathrm{~cm}^{-1}$.

$\mathrm{XP}$-spectrum of this film showed only one state in the Si2p region with a binding energy (BE) of $102.3 \mathrm{eV}$, which is characteristic for $\mathrm{Si}^{4+}$. The spectrum for the $01 \mathrm{~s}$ core level revealed two components centered at 531.3 and $529.8 \mathrm{eV}$. Again, the peak separation $(1.5 \mathrm{eV})$ and the 
intensity ratio ( 3:2) are very similar to those found for the $\mathrm{SiO}_{2.5} / \mathrm{Mo}(112)$ films $(1.3 \mathrm{eV}$ and 3:2, respectively), where the high $\mathrm{BE}$ signal is associated with the oxygen atoms forming the Si-O-Si bonds (henceforth referred to as $\mathrm{O}(\mathrm{Si})$ atoms), and the low $\mathrm{BE}$ signal corresponds to interfacial O species having bonds to the Mo substrate, i.e. to the Si-O-Mo linkages.


Figure 2. STM images (a-c) and IRAS spectra (d-f) of silica films grown on $\mathrm{Ru}(0001)$ at increasing Si coverage: $1 \mathrm{MLE}(\mathrm{a}, \mathrm{d}) ; 1.6 \mathrm{MLE}(\mathrm{b}, \mathrm{e})$; and $4 \operatorname{MLE}(\mathrm{c}, \mathrm{f})$.

Therefore, combined, these results provide compelling evidence that such a silica film on $\mathrm{Ru}(0001)$ is structurally very similar to the monolayer film on $\mathrm{Mo}(112)$ and consists of a honeycomb shaped network of tetrahedral Si-O linkages (see Fig. 1a), in which every Si makes one 
Si-O-Ru bond and three bridging Si-O-Si bonds. Henceforth we refer to the amount of deposited Si to form a closed monolayer film as one monolayer equivalent (1 MLE).

DFT calculations nicely support the proposed structure. It turned out, however, that among the several monolayer structures computed, only the film containing additional two oxygen atoms, directly adsorbed on $\mathrm{Ru}$ (henceforth referred to as $\mathrm{O}(\mathrm{Ru})$ atoms), per rectangular unit cell, is stable (see Fig. 3a). (Note, that the presence of additional $\mathrm{O}$ atoms on Mo also increased the stability of the monolayer films on $\mathrm{Mo}(112))$. The harmonic IRA spectrum simulated for the most stable monolayer structure shown in Fig. $3 c$ revealed the most intense IR-active mode at $\sim 1160 \mathrm{~cm}^{-1}$ which originates from the in-phase combination of asymmetric stretching vibrations of the Si-O-Ru linkages. The much weaker mode at $1076 \mathrm{~cm}^{-1}$ involves combinations of symmetric O-Si-O stretching vibrations. The bands at 820 and $677 \mathrm{~cm}^{-1}$ are the combinations of asymmetric stretching of Si-O-Ru linkages and O-Si-O bending modes. The positions and relative intensities of these calculated bands are in good agreement with the experimental data shown in Fig. 2d. ${ }^{[18]}$

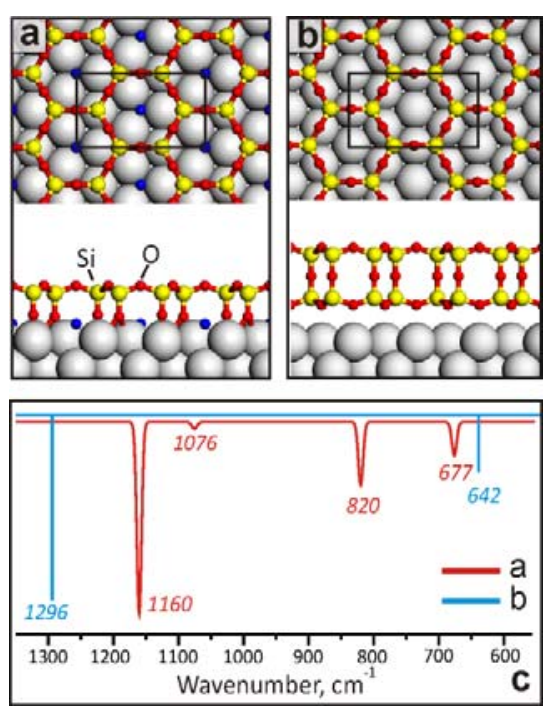

Figure 3. (a,b) Top and cross views of DFT-optimized monolayer (a) and bilayer (b) structures of the films grown on Ru(0001). Panel (c) shows harmonic IR spectra calculated for structures (a) and (b), respectively, to compare with the experimental spectra shown in Figs. 2(d,e).

Increasing Si coverage up to 2 MLE results in no change in the surface symmetry of the silica film (monitored by LEED), but in vibrational spectra, where very sharp and intense bands 
at 1300 and $692 \mathrm{~cm}^{-1}$ develop with concomitant disappearance of the bands associated with the monolayer structure (Fig. 2e). The STM images of such films revealed flat morphology, where wide terraces of underlying $\mathrm{Ru}(0001)$ can still be recognized (Fig. 2b). At intermediate Si coverages, extended domains and/or islands within the same terrace were separated by steps with apparent heights of $\sim 1.5$ and $\sim 5 \AA$ when measured with respect to small holes, presumably exposing the underlying O/Ru surface. The $1.5 \AA$ A-high steps can straightforwardly be assigned to the monolayer structure, whereas the $\sim 5 \AA$-high steps indicate formation of a multilayer silica film. High-resolution STM images of such domains showed again honeycomb-like structures with a $5.5 \AA$ A periodicity (inset in Fig. 2b), like in monolayer films (Fig. 2a), thus suggesting the formation of bilayer structures composed of two silica layers linked together through bridging $O$ atoms originally bonded to a metal support in the monolayer film (see scheme in Fig. 1b).

This model, shown in Fig. 3b, was tested by DFT including semi-empirical dispersion correction (DFT+D). The calculated adhesion energy of the bilayer silica film to $\mathrm{Ru}(0001)$ support was only about $3 \mathrm{~kJ} \mathrm{~mol}^{-1} \AA^{-2}$, with the main contribution coming from the dispersion term. The phonon spectrum, calculated for this structure, revealed two IR active modes (see Fig. 3c). The most intense mode at $1296 \mathrm{~cm}^{-1}$ is an in-phase combination of asymmetric Si-O-Si stretching vibrations of the Si-O-Si linkage between two layers. The second mode at $642 \mathrm{~cm}^{-1}$ is a combination of symmetric Si-O-Si stretching vibrations of Si-O-Si bonds nearly parallel to the surface. Not only the positions but also relative intensities of the vibrational bands are in very good agreement with the experimental results.

Thermal stability of these films was examined by XPS as a function of annealing temperature (see Fig. 4). It turned out that the $\mathrm{Si} 2 \mathrm{p}$ and $\mathrm{O} 1 \mathrm{~s}(\mathrm{Si})$ core levels shifted by about $0.8 \mathrm{eV}$ upon UHV annealing to $1150 \mathrm{~K}$, such that the energy separation between the Si2p and O1s(Si) peaks remained constant. Also the intensity and the width of these signals did not change. The valence band responded to the thermal treatments in the same manner as the core levels: Silica related features shifted by $\sim 0.8 \mathrm{eV}$. Note, that annealing did not cause any detectable changes in LEED and IRAS, indicating that the silica film maintains chemical composition and stoichiometry. In contrast, the O1s(Ru) peak did not shift, but the intensity decreased upon stepwise UHV annealing. 



Figure 4. Top panel: Si $2 p$ and O1s levels in XP-spectra of the bilayer silica films on Ru(0001) measured with the synchrotron light. Bottom panel: BE shifts of the respective core levels upon annealing in UHV at temperatures indicated. The spectra shift back upon re-oxidation. The inset shows the structure model with additional $\mathrm{O}(\mathrm{Ru})$ atoms, directly adsorbed on $\mathrm{Ru}(0001)$ surface underneath the bilayer film.

All these findings suggest that only the interfacial $\mathrm{O}(\mathrm{Ru})$ atoms between $\mathrm{SiO}_{2}$ and $\mathrm{Ru}(0001)$ desorbs upon UHV annealing, ultimately forming so-called "O-poor" films as compared to the "as prepared", "O-rich" films, the nomenclature we have used for silica films on Mo(112). The transition between the two states is fully reversible: The spectra shift back after sample re-oxidation.

Again, theoretical calculations nicely corroborated the experimental observations by finding gradual $\mathrm{BE}$ shifts upon increasing the number of the $\mathrm{O}(\mathrm{Ru})$ atoms in the unit cell. Oxygen adsorption on the $\mathrm{Ru}(0001)$ surface considerably lowers the adhesion energy. Certainly, this effect is connected to the repulsive interaction between the oxygen atoms of the silica film and the $\mathrm{O}(\mathrm{Ru})$ atoms. As a result the distance between the silica sheet and metal support increases. However, the additional calculations showed that the BEs shift is almost independent of the 
distance between the silica film and the metal substrate and is primarily related to the change of electronic properties of the metal substrate induced by $\mathrm{O}$ adsorption.

Therefore, the results show that the $\mathrm{SiO}_{2} / \mathrm{Ru}$ electronic states reversibly vary with the amounts of adsorbed oxygen underneath the silica film. In principle, this opens a possibility to tune electronic properties of oxide/metal systems without altering the thickness and structure of an oxide overlayer itself. ${ }^{[17,19-21]}$

Further increasing Si coverage up to 4 MLE results in smooth films, albeit not atomically flat (Fig. 2c). Unfortunately, atomic resolution could not be achieved on these films, as the STM imaging became unstable due to the poor electrical conductivity of the thick silica films. Nonetheless, as in the case of mono- and bilayer structures, substantial changes are observed by IRAS. As shown in Fig. 2f, a new band develops at $1257 \mathrm{~cm}^{-1}$ with a prominent shoulder at 1164 $\mathrm{cm}^{-1}$, while the 1300 and $694 \mathrm{~cm}^{-1}$ peaks strongly attenuate. The band centered at $1257 \mathrm{~cm}^{-1}$ is virtually identical to that previously observed on several nanometers thick silica films grown on Mo and Si single crystal supports. The bands at 1257 and $1164 \mathrm{~cm}^{-1}$ are assigned to the asymmetric longitudinal-optical vibration modes as in quartz-like compounds. It therefore appears that thicker films exhibit a three-dimensional network of $\left[\mathrm{SiO}_{4}\right]$ tetrahedral (see Fig. 1d) rather than the layered structure observed for mono- and bi-layer films. In this case, there is no unique termination of the film which therefore results in a relatively high surface corrugation (ca. 1 A) as measured by STM.

Summarizing this part, we realize that in contrast to Mo(112), which accommodates only monolayer well-ordered silica films, both mono- and bi-layer, depending on the Si coverage maybe grown on $\mathrm{Ru}(0001)$. The structure of the films can be straightforwardly identified by their IR spectra, as each structure shows a characteristic vibrational band, i.e., $\sim 1135 \mathrm{~cm}^{-1}$ for a monolayer film, $\sim 1300 \mathrm{~cm}^{-1}$ for the bilayer structures, and $\sim 1250 \mathrm{~cm}^{-1}$ for the bulk-like silica.

\section{Crystalline versus Vitreous Silica Films}

Depending on the preparation conditions, LEED patterns of the bilayer films may show both, $(2 \times 2)$ spots and a diffraction ring. The latter indicates the presence of randomly oriented rotational domains like in powders. Indeed, the respective STM images revealed domains of 
ordered structures coexisting with disordered structures, which could be identified as twodimensional, vitreous silica. Analysis of numerous bilayer films prepared under different conditions by varying annealing time, temperature, oxygen pressure, etc. showed that the rate of sample cooling after the high-temperature oxidation step as well as the initial amount of silicon play an important role in controlling film crystallinity. The atomic structure of crystalline and vitreous domains of the bilayer film was investigated using non-contact atomic force microscopy (nc-AFM) and STM. In Fig. 5a typical STM image is presented revealing neighboring crystalline and vitreous patches from left to right.



Figure 5. High resolution STM image of a typical silica bilayer film, where crystalline and vitreous phase are observed at the same sample spot $\left(\mathrm{V}_{\mathrm{S}}=2 \mathrm{~V}, \mathrm{I}_{\mathrm{T}}=100 \mathrm{pA}\right.$, scan area $\left.=10 \mathrm{~nm} \times 5 \mathrm{~nm}\right)$.

In 1932 William H. Zachariasen published his postulates on "The Atomic Arrangement in Glass". ${ }^{[1]}$ In his approach he has tried to propose two-dimensional analogs to the complex three-dimensional arrangements of polyhedral networks. 

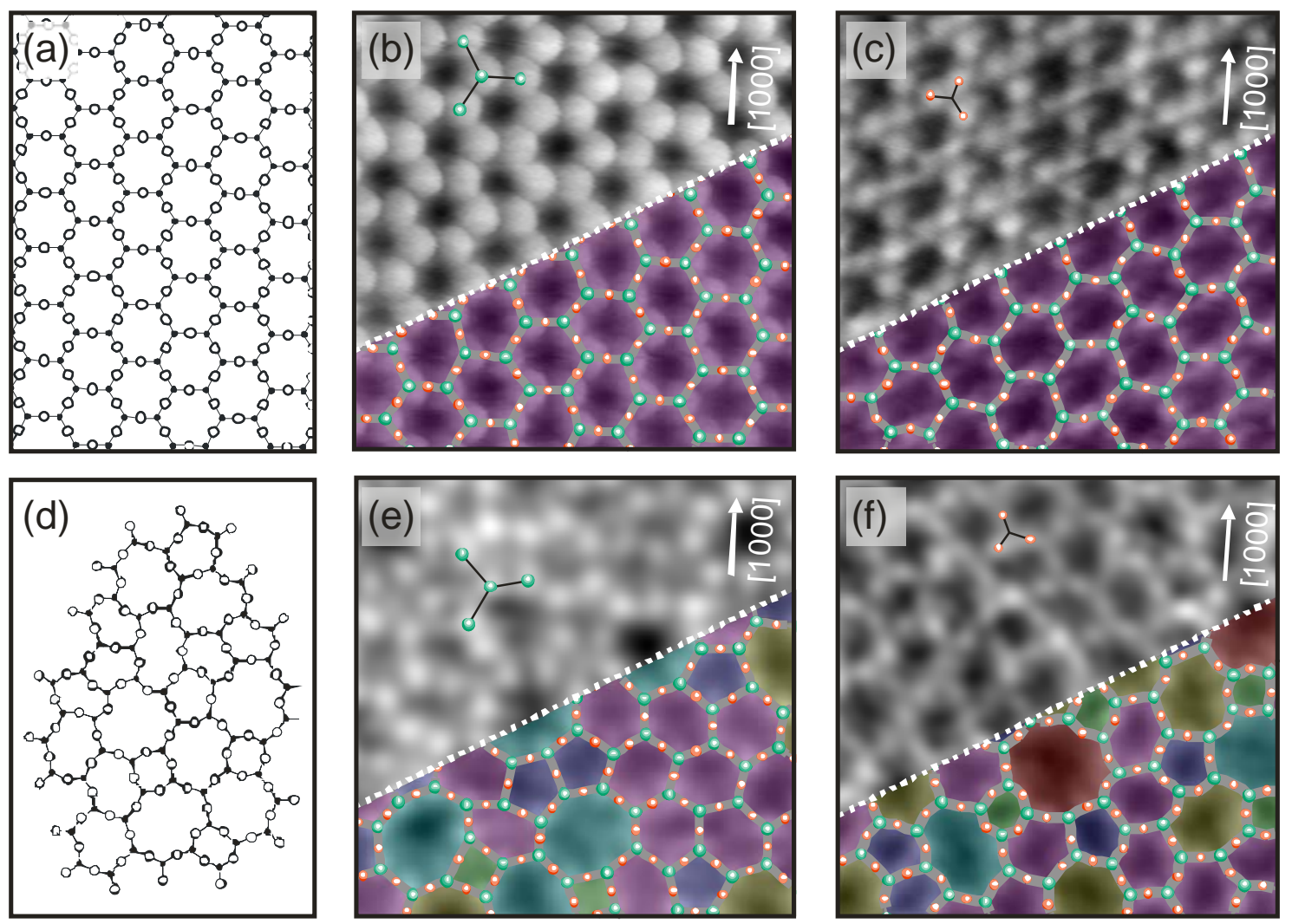

Figure 6. (a,d) Zachariasen's schemes of the atomic arrangement of a crystal (a) and a glass (d) (from [1]). $(b, c, e, f)$ Atomically resolved crystalline and vitreous regions of the thin silica film (the scan area of all images is $3.5 \mathrm{~nm} \times 3.5 \mathrm{~nm}$ ). (b) STM image of a crystalline area showing the positions of Si a toms (Vs := $3.0 \mathrm{~V}, \mathrm{~h}:=100 \mathrm{pA}$ ). (c) STM image of a crystal line patch showing the arrangement of $\mathrm{O}$ atoms (Vs $:=100$ $\mathrm{mV}, \mathrm{h}:=100 \mathrm{pA}$ ). (e) STM image of a vitreous area revealing the positions of $\mathrm{Si}$ a toms (Vs:= $2.0 \mathrm{~V}, \mathrm{~h}:=$ $50 \mathrm{pA}$ ). (f) STM image of a vitreous area showing the arrangement of $O$ atoms (Vs := $100 \mathrm{mV}, \mathrm{h}:=100$ pA). (b,c,e,f) Arrows indicate one crystallographic axis of the $\mathrm{Ru}(0001)$ substrate. Green balls represent the $\mathrm{Si}$ a toms, red balls the 0 atoms.

His ideas of such network structures are sketched in Fig. 6a and $6 \mathrm{~d}$ for the crystalline and vitreous arrangements respectively. The white circles represent the anions (in our case 0 ) and the black dots the cations (Si). Each black dot is surrounded by three white circles, forming the network building block for both phases. By changing the angle between these building blocks and linking the white circles to not more than two black dots allows to generate crystalline and vitreous two-dimensional network structures. The presented cut-outs in Fig. 6b-c and Fig. 6e-f are taken from STM images showing the atomic arrangement in both phases of the bilayer silica films. We were able to resolve the atomic positions of the topmost $\mathrm{O}$ in Fig. $6 \mathrm{~b}, \mathrm{~d}$ and Si sites in Fig. $6 \mathrm{c}$, f for both silica phases. The contrast in these STM images depends, of 
course, on the tunneling parameters, but even more on the configuration and chemical interaction in the tunneling junction. The STM images in Fig. 6 are also superimposed by atomic models (O red, Si green) providing the detailed ring morphology and additional color coding in order to highlight the different ring sizes. We have clearly assigned both Si and $\mathrm{O}$ atoms in the crystalline and vitreous structures.

In the crystalline phase obviously only one ring size is present, namely six membered rings, consisting of $6 \mathrm{Si}$ and $6 \mathrm{O}$ atoms. In the vitreous phase, pores ranging from four- to ninemembered rings can be seen. A detailed analysis of histogram plots has been performed. The histogram the most common ring has six members. A slight asymmetric distribution for more five- than seven-rings is observed in these plots. ${ }^{[9]}$ The ring size distribution can be analyzed in a log-normal plot. Thereby, the probability density function of the ring sizes follows a linear behavior in the log-normal scale, which is in line with theoretical models of two-dimensional triangle rafts. ${ }^{[22]}$ In a recent study we have looked at characteristic structural properties of crystalline and vitreous domains. A large amount coordinates has been derived from several high resolution nc-AFM and STM measurements. Atomic distances, orientations and angles were determined, comparing our results to experiments and simulations of bulk silica networks. It was observed that crystalline regions exhibit minor effects of disorder. This effect has been attributed to the flexible network structure. The evaluation of inter-atomic distances and angles showed good overall agreement to diffraction data and simulated networks of bulk silica. Surprisingly, also the extension of order is similar for the $2 \mathrm{D}$ and $3 \mathrm{D}$ vitreous structures. ${ }^{[11]}$ It becomes obvious that the silica bilayer film in its crystalline and vitreous domains is an ideal model system to study and understand various properties of complex network structures with the link to bulk glasses.

A useful way to compare results of scanning probe microscopic investigations with scattering data collected with $\mathrm{x}$-ray diffraction or neutron diffraction techniques on amorphous materials is to compute pair correlation functions (PCF).

Figure 7 shows pair distribution histograms for $\mathrm{Si}-\mathrm{O}, \mathrm{O}-\mathrm{O}$, and $\mathrm{Si}-\mathrm{Si}$ determined from scanning probe images. First peaks in all three distributions correspond to the respective nearest neighbor distances. The first Si-O peak is located at $156+-14$ pm, the $0-0$ peak at $258+-32$ 
pm, and the Si-Si peak at 296+-14 pm. The amorphous nature of the film is reflected in the peaks getting broader for larger distances. Good comparison with Total Pair Correlation Functions determined via x-ray- and neutron-diffraction in Figure 7. The short- and medium ranges are basically reproduced. The deviations are connected with the two-dimensional nature of the silica film. It is still surprising how close those distribution functions of three dimensional materials are to this vitreous silica film.

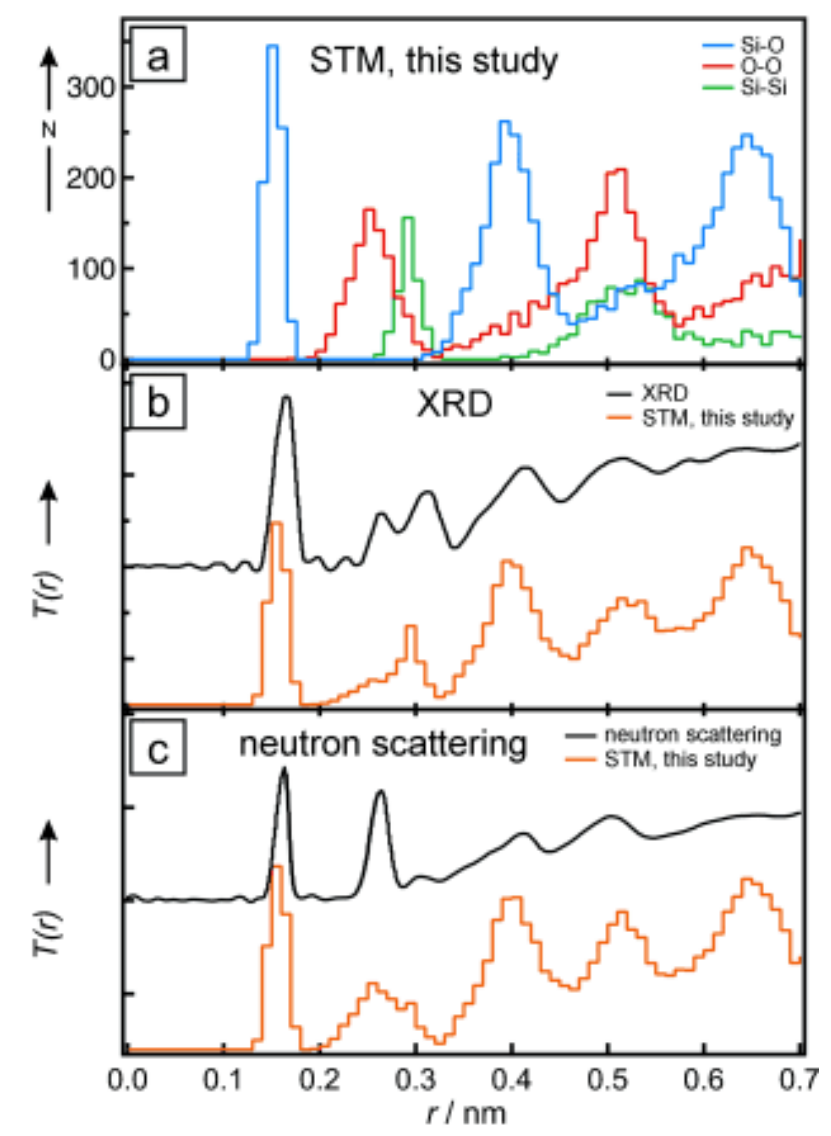

Figure 7: Pair correlation function. a) The pair distance histograms for $\mathrm{Si}-\mathrm{O}$ (blue), $\mathrm{O}-\mathrm{O}$ (red), and $\mathrm{Si}-\mathrm{Si}$ (green). b) Comparison of the total pair correlation function, $T_{\text {STM }}(r)$ (orange curve), with the PCF obtained from X-ray diffraction measurements on vitreous silica (black curve). ${ }^{[23]} \mathrm{c}$ ) Comparison of $T_{\text {STM }}(r)$ (orange curve) with results from neutron scattering on vitreous silica (black curve). ${ }^{[24]}$

Besides the analysis of the pure crystalline and vitreous phase, this model system allows addressing even structural transitions between them. The impact of visualizing such interface and the importance for generating a defined model for understanding the crystal-to-glass or the liquid-to-glass transition becomes clear. Here, in Fig. 8 an STM image with atomic resolution 
of such transition is shown. The image has been superimposed by its determined atomic positions in $7 \mathrm{~b}$ and color coded for the different ring sizes in $8 \mathrm{c}$.
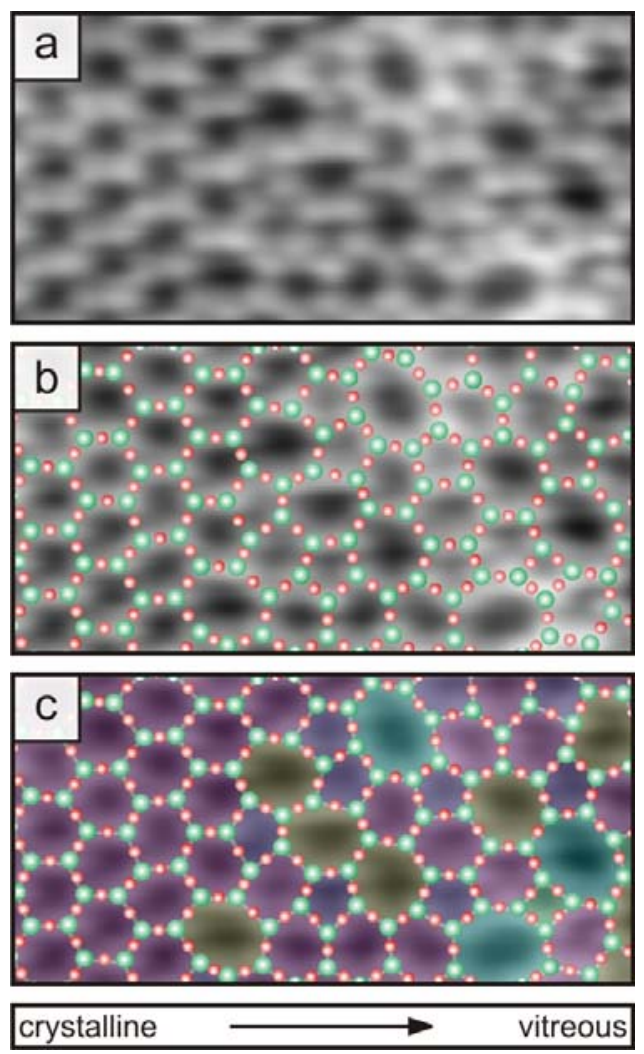

Figure 8. (a) STM image of an atomically resolved transition between a crystalline and vitreous domain in the bilayer silica film (cut-out from Fig. $5, \mathrm{VS}=2 \mathrm{~V}, \mathrm{IT}=100 \mathrm{pA}$, scan area $=5.0 \mathrm{~nm} \times 2.5 \mathrm{~nm}$ ). (b) The STM image, superimposed by the atomic model of the topmost layer (Si green, $O$ red). (c) Color coded ring sizes. The black arrow in the bar below indicates the direction and decay length of the interface from the crystalline to the vitreous area.

The atomically resolved coordinates allow determination of the local environment of nearest neighbor distances, characteristic angles and the progression of neighboring ring sizes inside of these transition regions. Diffractional methods do not allow for such a direct assignment, which is here only possible due to the local real space imaging capabilities of scanning probe microscopy techniques. A complete analysis, discussion, and the coordinates for such transition have been provided. In the region of the interface, typical building blocks of the vitreous phase are used and no other defects in terms of unsaturated bonds or different bonding arrangements are observed (see Fig. 8c). The decay length of this transition region is in the 
range of $1.6 \mathrm{~nm}$. In this interface area from the crystalline to the vitreous phase the evolution from six-membered to different rings starts off with five- and seven-membered rings followed by sizes of four, eight, and nine until the distribution of a typical vitreous ring network is

reached. ${ }^{[10]}$ The crystalline phase smoothly transforms into the vitreous area. Thereby, we have given a nice example where we have proven that this model system has the capabilities to study tetrahedral network structures at the atomic scale. But even more important is that it is a real model for the structural glass-transition due to its chemical composition and stoichiometry of $\mathrm{SiO}_{2}$.

\section{Concluding Remarks}

The investigation of flat thin silica films grown on single crystal metal supports has turned out to be a playground to tackle a number of interesting problems. They range from applied areas, regarding support materials for model studies in catalysis to the fundamental problem of investigating the crystal-glass transition at atomic resolution, a problem under study for more than 80 years. This short review reveals some of the details of the variety of structures formed and indicates a number of directions for future research. The study also opens up opportunities to look for model systems involving silica films in the area of catalysis. There are catalytic processes involving specific metals anchored to silica in polymerization of alkenes. The Phillips catalyst would be an example. This could then be studied comparing crystalline and vitreous support.

\section{Acknowledgements}

We thank all our co-workers, whose names appear in the cited papers, for their tremendous efforts. This work has been supported by Fonds der Chemischen Industrie, DFG, CoE UniCat, SFB 546. 


\section{References}

[1] W. H. Zachariasen J. Am. Chem. Soc. 1932, 54, 3841-3851.

[2] K. T. Queeney, M. K. Weldon, J. P. Chang, Y. J. Chabal, A. B. Gurevich, J. Sapjeta, R. L. Opila J. Appl. Phys. 2000, 87, 1322-1330.

[3] R. Zallen, The Physics of Amorphous Solids, John Wiley \& Sons, Inc., New York, 1983.

[4] J. Weissenrieder, S. Kaya, J.-L. Lu, H.-J. Gao, S. Shaikhutdinov, H.-J. Freund, M. Sierka, T. K. Todorova, J. Sauer Phys. Rev. Lett. 2005, 95, 076103.

[5] D. Loeffler, J. J. Uhlrich, M. Baron, B. Yang, X. Yu, L. Lichtenstein, L. Heinke, C. Büchner, M. Heyde, S. Shaikhutdinov, H. J. Freund, R. Wlstrokodarczyk, M. Sierka, J. Sauer Phys. Rev. Lett. 2010, 105, 146104.

[6] D. J. Stacchiola, M. Baron, S. Kaya, J. Weissenrieder, S. Shaikhutdinov, H.-J. Freund Appl. Phys. Lett. 2008, 92, 0119111-0119113.

[7] T. Schröder, M. Adelt, B. Richter, M. Naschitzki, M. Bäumer, H.-J. Freund Surf. Rev. Lett. 2000, 7, 714.

[8] X. Yu, B. Yang, J. A. Boscoboinik, S. Shaikhutdinov, H.-J. Freund Appl. Phys. Lett. 2012, 100, 151608.

[9] L. Lichtenstein, C. Büchner, B. Yang, S. Shaikhutdinov, M. Heyde, M. Sierka, R. Włodarczyk, J. Sauer, H.-J. Freund Angew. Chem. Int. Ed. 2012, 51, 404-407.

[10] L. Lichtenstein, M. Heyde, H.-J. Freund Phys. Rev. Lett. 2012, in press.

[11] L. Lichtenstein, M. Heyde, H.-J. Freund J. Phys. Chem. C. 2012, submitted.

[12] W. Raberg, K. Wandelt Appl. Phys. A: Mater. Sci. Process. 1998, 66, 1143-1146.

[13] J. F. Poggemann, G. Heide, G. H. Frischat J. Non-Cryst. Solids. 2003, 326-327, 15-20.

[14] G. H. Frischat, J. F. Poggemann, G. Heide J. Non-Cryst. Solids. 2004, 345-346, 197-202.

[15] W. Raberg, A. H. Ostadrahimi, T. Kayser, K. Wandelt J. Non-Cryst. Solids. 2005, 351, 1089-1096.

[16] R. Włodarczyk, M. Sierka, J. Sauer, D. Löffler, J. J. Uhlrich, X. Yu, B. Yang, I. M. N. Groot, S. Shaikhutdinov, H. J. Freund Phys. Rev. B. 2012, 85, 085403.

[17] U. Martinez, J.-F. Jerratsch, N. Nilius, L. Giordano, G. Pacchioni, H.-J. Freund Phys. Rev. Lett. 2009, $103,056801$.

[18] L. Lichtenstein, M. Heyde, S. Ulrich, N. Nilius, H.-J. Freund J. Phys. Cond. Matt. . 2012, in press.

[19] S. Ulrich, N. Nilius, H.-J. Freund, U. Martinez, L. Giordano, G. Pacchioni Phys. Rev. Lett. 2009, 102, 016102.

[20] S. Ulrich, N. Nilius, H.-J. Freund, U. Martinez, L. Giordano, G. Pacchioni ChemPhysChem. 2008, 9, 1367-1370.

[21] M. Baron, D. Stacchiola, S. Ulrich, N. Nilius, S. Shaikhutdinov, H. J. Freund, U. Martinez, L. Giordano, G. Pacchioni J. Phys. Chem. C. 2008, 112, 3405-3409.

[22] J. F. Shackelford, B. D. Brown J. Non-Cryst. Solids. 1981, 44, 379-382.

[23] R. L. Mozzi, B. E. Warren J. Appl. Crystallogr. 1969, 2, 164-172.

[24] D. I. Grimley, A. C. Wright, R. N. Sinclair J. Non-Cryst. Solids. 1990, 119, 49-64. 